Vol 12, Issue 10, 2019

\title{
NIGELLA SATIVA OIL AS AN ADD-ON IN THE CONSERVATIVE MANAGEMENT OF DIABETIC NEPHROPATHY: A NOVEL SYNERGISTIC APPROACH
}

\author{
MOHD ASHRAF ALAM ${ }^{1 *}$, MOHAMMAD NASIRUDDIN ${ }^{1}$, SHAHZAD F HAQUE ${ }^{2}$, RAHAT A KHAN ${ }^{1}$ \\ ${ }^{1}$ Department of Pharmacology, Jawaharlal Nehru Medical College and Hospital, Aligarh Muslim University, Aligarh, Uttar Pradesh, India. \\ ${ }^{2}$ Department of Medicine, Jawaharlal Nehru Medical College and Hospital, Aligarh Muslim University, Aligarh, Uttar Pradesh, India. \\ Email: dr.mashrafalam@gmail.com
}

Received: 18 July 2019, Revised and Accepted: 04 September 2019

ABSTRACT

Objective: The objective of the study was to evaluate efficacy and safety Nigella sativa oil as an add-on therapy in addition to alpha-keto analog of essential amino acids in patients diabetic nephropathy (DN).

Methods: The study was conducted in a tertiary care center of North India on patients of DN. It was a prospective, comparative, and open study. Patients were randomly divided into two interventional groups. Group I (control) received conservative management of chronic kidney disease consisting of alpha-keto analog (600 mg tab. 3 times a day), while Group II (test) received conservative management along with alpha-keto analog and $N$. sativa oil ( $2.5 \mathrm{ml}$, per orally, once daily) for 12 weeks. Hemogram and renal function, serum electrolytes tests were done, and adverse events were recorded at baseline, 4,8 , and 12 weeks of treatment.

Results: After 12 weeks of treatment, there was a marked improvement in clinical features and biochemical parameters in both the control and test groups, but it was more statistically significant in the test group as compared to control group especially in renal function ( $\mathrm{p} \leq 0.05$ ). Both groups showed steady improvement in the biochemical parameters as compared to their pre-treated values which were more marked in $N$. sativa oil supplemented group. There was a reduction in blood glucose, blood urea, serum creatinine, and 24-h total urine protein, and an increase in hemoglobin, 24-h total urine volume, and glomerular filtration rate.

Conclusion: $N$. sativa oil supplementation along with alpha-keto analog is more efficacious and safer in delaying the progression of disease patients of DN.

Keywords: End-stage renal disease, Diabetic nephropathy, Diabetes mellites, Chronic kidney disease, Glomerular filtration rate, Alpha-keto analog, Nigella sativa oil.

(C) 2019 The Authors. Published by Innovare Academic Sciences Pvt Ltd. This is an open access article under the CC BY license (http://creativecommons. org/licenses/by/4. 0/) DOI: http://dx.doi.org/10.22159/ajpcr.2019.v12i10.34985

\section{INTRODUCTION}

The prevalence of type-2 diabetes mellitus (T2DM) is rampant and is increasing in all age groups. According to the database of the World Health Organization, the global prevalence of diabetes in adults above 18 years was estimated to be $9 \%$ in 2014 . It has been estimated that 1.5 million deaths were directly caused by diabetes in 2012, and more than $80 \%$ of them were in low- and middle-income countries. Diabetes is projected to be the seventh leading cause of death by 2030 [1]. The total expenditure on diabetes in 2017 was estimated to be at least USD 727 billion (12\% of total expenses on health) [2]. India occupies the second position, after China, in the global list of countries with the highest prevalence of diabetes, among which the incidence of DN is on rising [1].

Persistent hyperglycemia leads to dyslipidemia in type 2 diabetic nephropathy (DN). Lipids may induce both glomerular and tubulointerstitial injury through inflammatory mediators, reactive oxygen species (ROS) and through hemodynamic changes $[3,4]$. Immune-inflammatory disturbances in type 2 diabetics have a deep-rooted association with cell-mediated changes, inappropriate T-lymphocyte function, and low-grade inflammatory status, which further the increased production of oxidative stress markers associated with type 2 DN. This inflammatory and oxidative stress are both deeply inter-related and are known to play a key role in diabetic vascular complications leading to end-stage renal disease (ESRD) [5].
The ideal treatment for ESRD is renal replacement therapy (RRT) which includes renal transplantation and maintenance dialysis. Even though renal transplant is the most preferred choice being, cost effective and offers a better quality of life but still, only a segment of Indian population can afford to have it, so conservative management is essential to prevent chronic kidney disease (CKD) [6].

$\alpha$ - keto analogs of essential amino acids are nitrogen-free analogs of essential amino acids. Keto-amino acids delay the progression of renal insufficiency. They are being used in DN since they have good glycaemic control, thereby improving insulin sensitivity and reducing hyperinsulinemia [7]. When $\alpha$-Keto amino acid is administered with low protein diet $(0.6 \mathrm{~g} / \mathrm{kg} /$ day $)$ or very low protein diet $(0.3 \mathrm{~g} / \mathrm{kg} /$ day), there is improved nitrogen balance and decreased urea accumulation in the body [8].

Nigella sativa role has been established as nephroprotective and antihyperglycemic in T2DM patients [9]. It has been proved that $N$. sativa oil improved renal function, liver function, fasting blood glucose (FbG), and glycated hemoglobin ( $\mathrm{Hb}$ ) level as well as having anti-inflammatory properties [10].

$\alpha$-keto analog of essential amino acids (KAA) is now being given as a standard treatment protocol in patients of DN; also, N. sativa oil has established itself strongly as having nephroprotective [9] effects. This study was done to see the efficacy and safety profile of $N$. sativa oil when given as an add-on in DN patients on standard regimen taking alphaketo analog. 


\section{METHODS}

The present study was conducted in patients of DN attending renal clinic or admitted to the hospital ward of a tertiary care center of North India from February-2016 to August-2017. It was a prospective, randomized, open-label, and parallel-group study which was approved by the Institutional Ethics Committee. This study was enlisted with Clinical Trials Registry-India (CTRI) with a registration number CTRI/2018/01/011429. All the patients gave written, informed consent voluntarily before registering for the study. The diagnosis of DN was made on the basis of detailed medical history, physical examination, and investigations (renal function test [RFT] and ultrasonogram).

\section{Inclusion criteria}

Patients with CKD (stage 3-4) and age 30-60 years of either gender were included in the study.

\section{Exclusion criteria}

Patients that were excluded from the study comprised pregnant females, patients undergoing dialysis, psychotic patients, immunocompromised or having severe renal pathology such as malignancy were excluded from the study.

\section{Study design}

A total of 80 patients were enrolled overall, of which 70 patients were met the inclusion criteria and were randomly divided into two groups of 35 patients each with the help of table designed by random allocation software in a ratio of 1:1. 8 patients (five patients of Group I, and three patients of Group II) failed to report on subsequent visits, and they were excluded from the study. Out of the remaining 62 patients, 30 patients were of Group I (control group) receiving KAA ( $600 \mathrm{mg}$ tablet), 3 times a day along with conservative management, and 32 patients were of Group II (test group) taking $N$. sativa oil ( $2.5 \mathrm{ml}$ once daily) and KAA in addition to conservative management. Registered patients after final diagnosis, applying inclusion, and exclusion criteria, were comprised in the study. Conservative management included (telmisartan, torsemide, iron, calcium, Vitamin D3, erythropoietin, and insulin in case of diabetic patients) of CKD. Both groups received treatment for 12 weeks. $\alpha$-keto analog tablets (KETOLOG) were of Claris Pharmaceuticals Company. $N$ sativa oil of $100 \%$ purity this study was commercially available procured from local market under brand name "Kalonji oil" from Mohammedia Products, Hyderabad, India (GMP certified company). The drugs above were procured from same pharmaceutical company throughout the study.

All the enrolled patients were regularly followed at 4, 8, and 12 weeks with following investigations:

All the enrolled patients were regularly followed up, with hemogram, renal functions test, and serum electrolytes at baseline, 4, 8, and 12 weeks of treatment.

\section{Safety assessments}

All adverse events experienced by a patient or observed by the investigator were recorded at each visit. Adverse drug reactions (ADRs) causality assessment was done using Naranjo Scale [10] and severity assessment by modified Hartwig and Siegel scale [11]. Physical examination and routine investigations were performed at the start of therapy and each visit. Additional laboratory test such as liver function tests and lipid profile test was done at start and end of study. All the ADRs were reported to the ADR monitoring center of the institute.

\section{Statistical analysis}

Statistical analysis was done using SPSS-23 software, and charts were prepared using Microsoft Excel 2016. For descriptive statistics; frequency, percentage, mean \pm standard deviation, and graphs were used to present the study result. Intra and intergroup analysis of the control and test groups was performed using t-test and repeated measures analysis of variance (rANOVA) test. Post hoc analysis was performed by post hoc Dunnett's test. $\mathrm{p}<0.05$ was considered statistically significant.

\section{RESULTS}

The maximum number of patients was in the age group of 30-60 years in both groups. Group I included 30 (11 males, and 19 females) patients and mean age was $47.2 \pm 14.67$ years (range $20-60$ years) while Group II included 32 (18 males, and 14 females) patients and mean age was $47.91 \pm 13.52$ (range $20-60$ years). With respect to age no significant difference observed between controls and test DN groups. The number of females was more than that of males in the control population (Table 1). In control and test group patients were also accessed for type of diet, smoking, and alcohol intake Fig. 1a.

There was neither mortality nor anyone required dialysis in either group. According to estimated glomerular filtration rate (eGFR) $\left(\mathrm{ml} / \mathrm{min} / 1.73 \mathrm{~m}^{2}\right)$, nine patients belonged to Stage 3 (3 and 6 in Groups I and II, respectively) and 53 patients Stage 4 CKD ( 27 and 26 in Groups I and II, respectively) (Fig. 1b). In Group I out of 30 patients, 10 patients were also having hypertension, and in Group II 8 patients were hypertensive.

The distribution of baseline demographic and biochemical data in the control group and test group is depicted in Table 2 and Fig. 2. In both control and test groups, no significant difference with respect to age is observed. In control group, females were more as compared to males. The rest parameters were comparable at baseline.

The different signs and symptoms observed in the patients at the start of the treatment were: Nausea, vomiting, weight loss, weakness, pruritus, headache, edema over the body, oliguria, burning during micturition, fever, dyspnea, anorexia, anemia, and hypertension. The clinical features were almost similar among the groups at baseline. The clinical features improved gradually and progressively in both

Table 1: Depicting the number of patients in control and test group with male and female ratio. Control group has more females in comparison to test group

\begin{tabular}{lllll}
\hline S. No. & Group & $\begin{array}{l}\text { Number of } \\
\text { patients }\end{array}$ & $\begin{array}{l}\text { Males } \\
\text { /females }\end{array}$ & Percentage \\
\hline 1. & I - Control (KAA) & 30 & $11 \mathrm{M} / 19 \mathrm{~F}$ & 48.38 \\
2. & $\begin{array}{l}\text { II - Test (N. sativa } \\
\text { oil+KAA) }\end{array}$ & 32 & $18 \mathrm{M} / 14 \mathrm{~F}$ & 51.62 \\
Total & & 62 & $29 \mathrm{M} / 33 \mathrm{~F}$ & 100.00 \\
\hline
\end{tabular}

KAA: $\alpha$-keto analog of essential amino acids, $N$. sativa: Nigella sativa

Table 2: Baseline demographic and biochemical parameters in controls and test group in patients of diabetic nephropathy. At baseline, the values were comparable in both the control and test groups, signifying a homogeneity in population in both groups. In control group, females were more as compared to males

\begin{tabular}{lll}
\hline Variables & Control group (n=30) & Test group (n=32) \\
\hline Age (years) & $47.2 \pm 14.67$ & $47.91 \pm 13.52$ \\
Sex & $11 \mathrm{M} / 19 \mathrm{~F}$ & $18 \mathrm{M} / 14 \mathrm{~F}$ \\
Hemoglobin & $8.27 \pm 1.34$ & $8.95 \pm 1.51$ \\
Systolic BP (mmHg) & $126.23 \pm 8.32$ & $132.5 \pm 11.72$ \\
Diastolic & $82.88 \pm 6.88$ & $84.32 \pm 11.66$ \\
BP (mmHg) & & \\
Blood sugar F & $98.76 \pm 10.97$ & $102.53 \pm 21.26$ \\
Blood sugar PP & $124.09 \pm 14.02$ & $132.84 \pm 30.08$ \\
HbA1c & $8.23 \pm 4.78$ & $9.42 \pm 5.22$ \\
Serum & $2.91 \pm 0.74$ & $2.85 \pm 0.69$ \\
creatinine (mg/dL) & & \\
Urea (mmol/24 h) & $84.47 \pm 26.11$ & $82.85 \pm 15.96$ \\
eGFR & $21.36 \pm 6.66$ & $22.37 \pm 6.85$ \\
TUP & $2.79 \pm 1.19$ & $2.68 \pm 0.89$ \\
\hline
\end{tabular}

*Significant at $\mathrm{p}<0.05$, n: Number of patients. BP: Blood pressure,

HbA1c: Glycated hemoglobin, eGFR: Estimated glomerular filtration rate, TUP: Total urine protein 


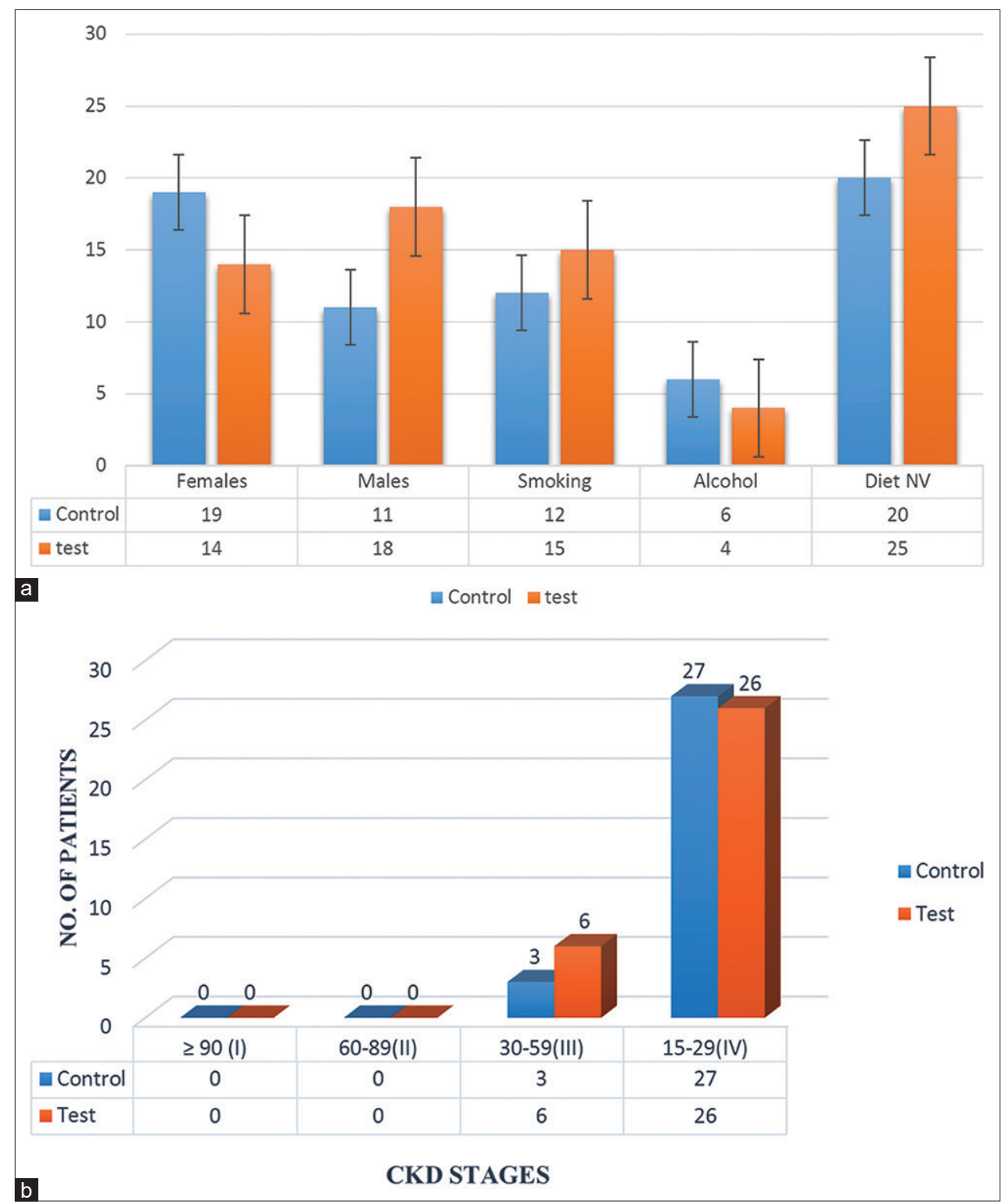

Fig. 1: (a) Demographic data of patients of diabetic nephropathy in control and test groups. (b) No. of patients in different stages of chronic kidney disease in control and test groups

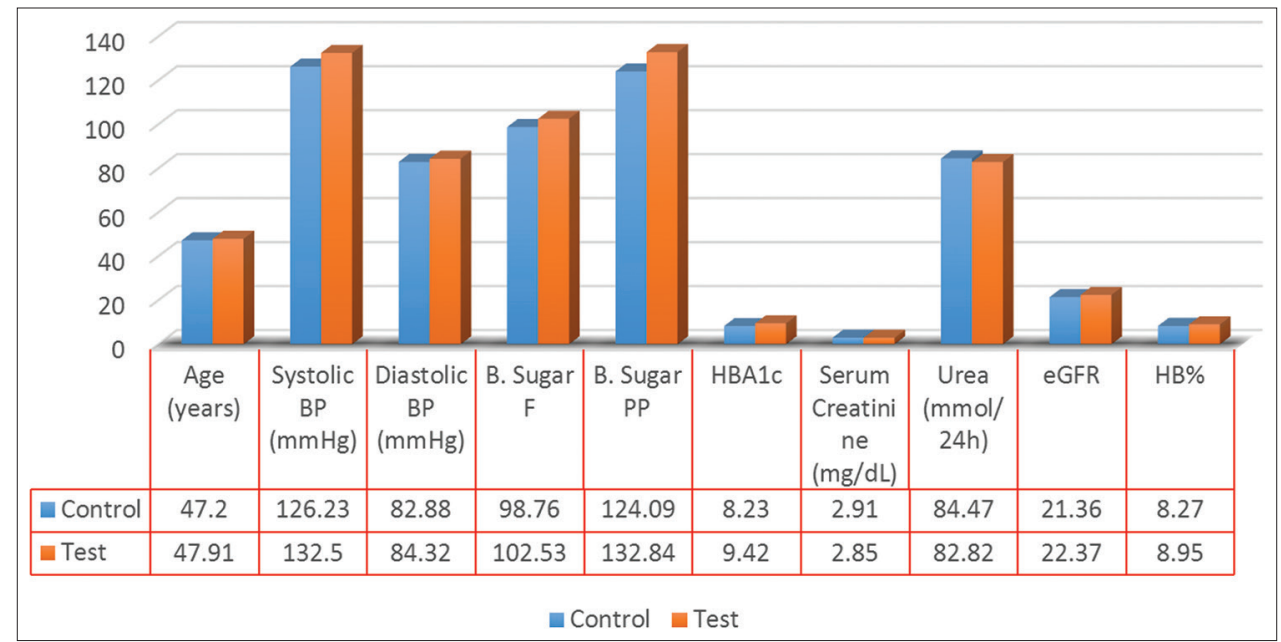

Fig. 2: Baseline demographic and biochemical parameters in controls and test group in patients of diabetic nephropathy. At baseline, the values were comparable in both the control and test groups, signifying a homogeneity in population in both groups. In control group, females were more as compared to males 
the groups after 12 weeks of treatment, but it was more significant in $N$. sativa oil-treated group Table 3 and Fig. 3 a.

We observed a highly significant $(\mathrm{p}<0.001)$ improvement in the $\mathrm{Hb} \%$ in both the groups, as compared to their baseline values at the end of 12 weeks. This improvement was also significant between the group's comparison at 4 weeks and 8 weeks. However, in the case of systolic and diastolic pressure, both the groups were comparable at baseline and the end of 12 weeks. The hypertensive patients were on medications for blood pressure, and their blood pressure was controlled, except for two patients who reported with high systolic blood pressure in the test group. In case of $\mathrm{FbG}$ and postprandial glucose, we observed a highly significant $(\mathrm{p}<0.001)$ improvement in both the groups, as compared to their baseline values at the end of 12 weeks. However, intergroup comparison showed insignificant $(p>0.05)$ change.

In Renal Functions, from the data depicted in Table 3 and Fig. $3 b$ we can observe that in blood urea estimation, a significant $(\mathrm{p}<0.001)$ improvement was observed within the control as well as test group, when comparing their values from baseline to end of 12 weeks. This change was also significant $(\mathrm{p}<0.001)$ between groups comparison, at 4,8 , and 12 weeks. Serum creatinine also showed a highly significant $(\mathrm{p}<0.001)$ progressive improvement in both the control and test groups from baseline to 12 weeks. However, when the groups were compared that the results were significant $(\mathrm{p}<0.05)$ at 4 and 8 weeks and highly significant $(\mathrm{p}<0.001)$ at 12 weeks. The results of GFR estimation within the groups comparison from baseline to 12 weeks were highly significant $(\mathrm{p}<0.001)$, whereas in between groups comparison the results were significant $(\mathrm{p}<0.05)$ at 4 weeks, very significant $(\mathrm{p}<0.01)$ at 8 weeks, and highly significant $(\mathrm{p}<0.001)$ at 12 weeks. We have also observed a highly significant $(\mathrm{p}<0.001)$ decline in total urine protein (TUP) in both the groups. However, in between groups comparison the results were significant $(\mathrm{p}<0.05)$ at 8 weeks and very significant $(\mathrm{p}<0.01)$ at 12 weeks.

According to modified Hartwig and Siegel scale, the ADRs were mild to moderate (no hospitalization, no change of therapy, and no additional treatment) in severity in both control and the test group. No adverse event of acute onset (within $60 \mathrm{~min}$ ) was seen. On Naranjo's Scale, the ADRs were possible (score=1-4) in 2 cases in the control group and 5 cases in test group, and probable (score=5-8) in 1 case with control group and 1 case in the test group (Table 4).

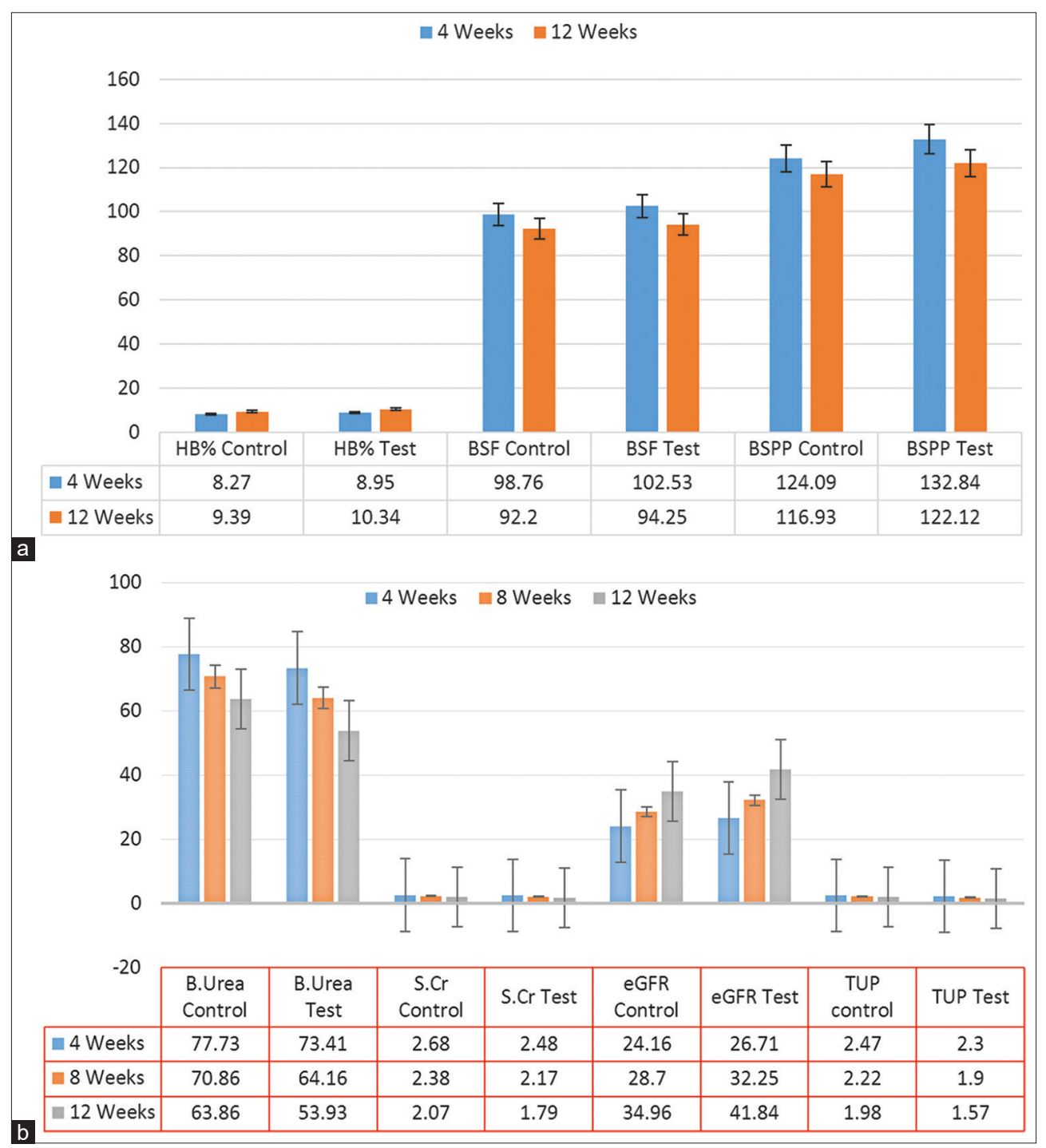

Fig. 3: (a) Biochemical parameters at the end of 4 weeks and 12 weeks in control and test groups in patients of diabetic nephropathy. (b) Change in renal functions at the end of 4, 8, and 12 weeks in patients of diabetic nephropathy in control and test groups. We see a significant decrease in the test groups as compared to the control in serum urea, serum creatinine, total urine protein, and estimated glomerular filtration rate 
Table 3: Biochemical parameters in at baseline 4, 8, and 12 weeks in control and test groups patients of diabetic nephropathy

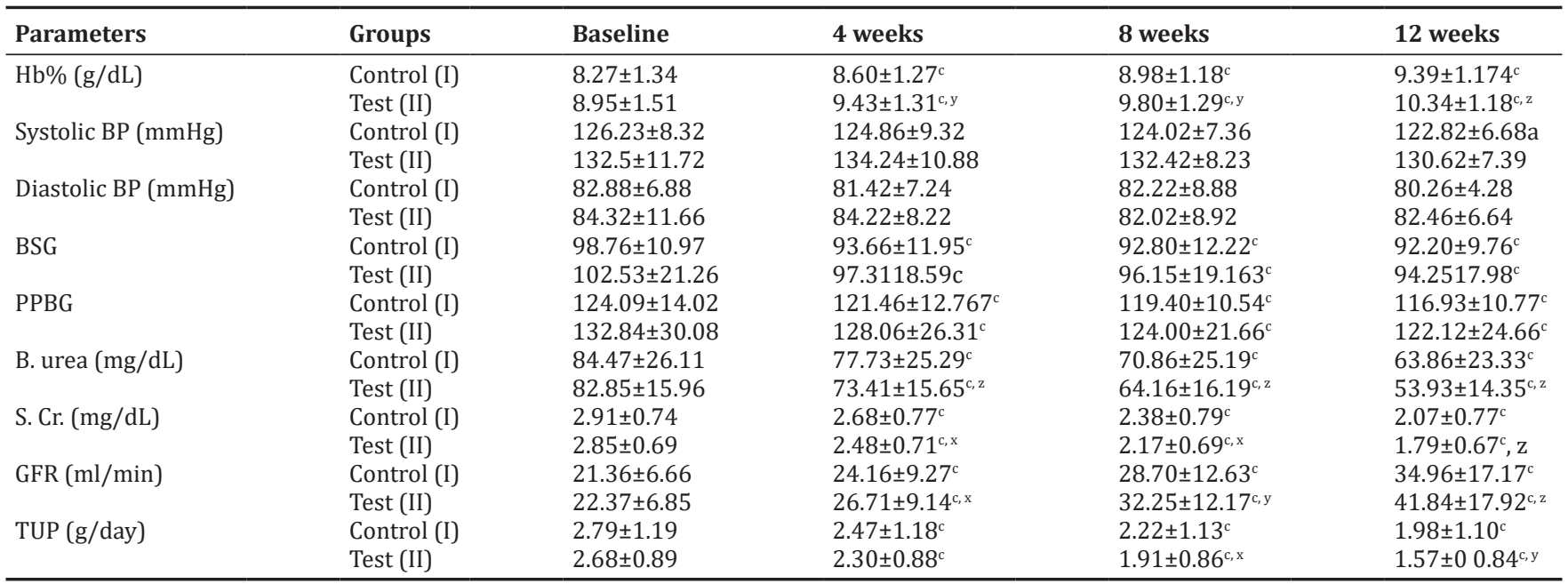

Values are mean $\pm \mathrm{SD}, \mathrm{p}<0.05$ was considered statistically significant, within-group comparison: ${ }^{\mathrm{a}} \mathrm{p}<0.05,{ }^{\mathrm{b}} \mathrm{p}<0.01$, ${ }^{\mathrm{c}} \mathrm{p}<0.001$. Between groups comparison: ${ }^{\mathrm{x}} \mathrm{p}<0.05$, ${ }_{\mathrm{y}}^{\mathrm{p}}<0.01 .{ }^{\mathrm{z}} \mathrm{p}<0.001$. Hb\%: Hemoglobin percent, B. urea: Blood urea, S. Cr.: Serum creatinine, FbG: Fasting blood glucose, PPBG: Postprandial blood glucose, TUP: $24 \mathrm{~h}$ total urinary protein, GFR: Glomerular filtration rate. For Group 1 ( $n=30$ patients) and Group 2 ( $n=32$ patients), SD: Standard deviation

Table 4: Comparison of ADRs due to KAA and KAA+N. sativa oil

\begin{tabular}{llll}
\hline S. No. & ADR recorded & KAA $(\mathbf{n}=\mathbf{3 0})$ & KAA+nigella oil $(\mathbf{n}=\mathbf{3 2})$ \\
\hline 1. & Nausea & 1 & 1 \\
2. & Vomiting & 0 & 2 \\
3. & Diarrhea & 0 & 1 \\
4. & Excessive & 1 & 1 \\
& thirst & & \\
5. & Rashes & 1 & 0 \\
6. & Altered taste & 0 & 1 \\
\hline
\end{tabular}

ADRs: Adverse drug reactions, KAA: $\alpha$-keto analog of essential amino acids, N. sativa: Nigella sativa

ADRs in control and test groups, where the most common ADR reported in the test group is vomiting, probably due to non-palatable taste of the N. sativa oil.

\begin{tabular}{|c|c|c|c|c|}
\hline \multicolumn{5}{|l|}{ Correlation table } \\
\hline \multirow[t]{2}{*}{ Parameters } & \multicolumn{2}{|c|}{ Control group } & \multicolumn{2}{|c|}{ Test group } \\
\hline & Baseline & 12 Weeks & Baseline & 12 Weeks \\
\hline Age versus eGFR & 0.158 & 0.147 & 0.144 & 0.137 \\
\hline $\begin{array}{l}\text { Hemoglobin \% } \\
\text { versus serum urea }\end{array}$ & -0.300 & -0.325 & 0.088 & 0.135 \\
\hline $\begin{array}{l}\text { Hemoglobin \% } \\
\text { versus eGFR }\end{array}$ & 0.245 & 0.258 & -0.011 & -0.171 \\
\hline Urea versus eGFR & $-0.469 *$ & $-0.531^{* *}$ & $-0.453^{*}$ & $-0.592^{* *}$ \\
\hline $\begin{array}{l}\text { Urea versus } \\
\text { creatinine }\end{array}$ & $0.560^{* *}$ & $0.503^{* *}$ & $0.401^{* *}$ & $0.437^{* *}$ \\
\hline
\end{tabular}

${ }^{* *}$ Correlation is significant at the $\mathrm{p}<0.01$ level (two-tailed). ${ }^{*}$ Correlation is significant at the $\mathrm{p}<0.05$ level (two-tailed)

\section{DISCUSSION}

Type 2 DN is a multifactorial and multidimensional and one of the most prevalent complications of DM worldwide, which accounts for nearly $44 \%$ of new cases [11]. Type 2 DM gradually affects the kidneys leading to which is the final stage of CKD. The diabetic prevalence has extended rampant magnitudes worldwide and is expected to cross 350 million people by the year 2035 [12]. The multi-risk factors for type 2 DN progression comprises a triad of genetic, environmental, and metabolic factors such as blood pressure, blood lipids, glycemic control, smoking, duration of diabetes, inflammatory changes, glomerular hypertension, and oxidative stress.
Long-standing hyperglycemia may directly increase the mesangial cell glucose concentration leading to mesangial expansion and injury. Initially, the glomerular mesangium expands by cell proliferation, followed by cell hypertrophy. This expansion is stimulated by an increase in mesangial stretch and pressure exerted by high glucose levels. Hyperglycemia aggravates glomerular lesions, and metabolic effects of long-standing hyperglycemia lead to advanced glycation end products (AGE) production which binds to collagen thereby leading to renal complications. AGE containing protein increases protein kinase $\mathrm{C}$ and transforming growth factor beta, which plays an important role in mesangial cell proliferation, differentiation, and cell apoptosis [13-16].

Conservative management plays a key role in DN in precluding ESRD, though it only delays the progressive decline of renal functions and offers symptomatic relief. Hence, novel modalities of treatment are being looked on, which can halt injury to nephron, as well as delay the progress to ESRD. Hence, treatment modalities are being which can cease nephron damage, defer the development of ESRD and are cost effective. Due to steep rise in the prevalence of diabetes and hypertension globally, CKD has emerged as a leading chronic disease worldwide $[17,18]$. CKD tends to cause premature morbidity, mortality, and hampers standard of life both economically and healthwise. The gold standard treatment for CKD-ESRD is RRT which includes renal transplantation and maintenance dialysis. There are about 5500 Dialysis Centers in India and over $90 \%$ of which are in the private sector [19]. In India, the monthly cost of the common dialysis prescriptions is approximately Rs. 29,852 (USD 609) [20]. Patients often cut down on dialysis frequency for economic reasons. Frequent and often long-term hospitalizations add to the financial burden [21].

In the present study, the different clinical parameters observed in the patients were: Nausea, vomiting, weight loss, pruritus, headache, hypertension, swelling over body, oliguria, burning during micturition, and anemia. The clinical features were in conformity with the symptomatology present in CKD patients described in literature. There was improvement in clinical features in both groups after 12 weeks of treatment. However, pronounced improvement was observed in test group especially in relation to $\mathrm{Hb}$ percent $(\mathrm{Hb} \%)$ and RFT parameters. We observed a progressive increase in $\mathrm{Hb} \%$ in both groups as compared to the baseline values. Yet, the results in the $N$. sativa supplemented group were more significant when comparison between the groups was made at the end of 12 weeks. This showed that $N$. sativa oil supplementation along with KAA produce more significant effect on $\mathrm{Hb} \%$. From the earlier studies, it has been proved 
that KAA causes significant stimulation of globin synthesis and thereby increases the hemoglobin level in patients of CKD [14]. It has also been reported from the previous studies that daily administration of $N$. sativa oil at a dose of $1 \mathrm{ml} / \mathrm{kg}$ body weight in rats increased $\mathrm{Hb} \%(\mathrm{mg} / \mathrm{dl})$ significantly $(\mathrm{p}<0.01)$ from $13.12 \pm 1.45$ to $15.4 \pm 0.64$ after 12 weeks of treatment [15]. Increase HB\% after supplementation of $N$. sativa oil might also have increased the $\mathrm{HB} \%$ in a similar giving an add-on benefit to KAA.

Interestingly, we observed a significant $(\mathrm{p}<0.001)$ reduction in blood urea levels in both the groups from baseline to 12 weeks. In addition, we also found that when comparing between groups, the results were highly significant $(\mathrm{p}<0.001)$ at 4,8 , and 12 weeks thereby indicating that test group showed better result as compared to control. These findings are in accordance with previous studies which showed that blood urea level decreased significantly $(\mathrm{p}<0.001)$ in patients of T2DM treated with $N$. sativa tea hot water extract as $5 \mathrm{~g}$ /day for 6 months [9]. Similar results were shown in the study by Khan et al. [22], where significant reduction was seen in blood glucose, blood urea, serum creatinine, and $24 \mathrm{~h}$ TUP along with increase in $\mathrm{Hb}, 24 \mathrm{~h}$ total urine volume, and GFR. It was postulated that supplementation of thymoquinone, an active component of $N$. sativa oil, prevents the development of renal failure by a mechanism related to decline oxidative stress by preserving the activity of antioxidant enzymes [23].

A 24-h TUP declined steadily in both groups, and the reduction was significant $(p<0.001)$ as compared to their baseline values at the end of 12 weeks. These findings were in accordance with the studies which demonstrated that $N$. sativa oil supplementation in patients of CKD leads to reduction in proteinuria and other RFT parameters [24].

GFR estimation the results within the groups comparison from baseline to 12 weeks were highly significant $(p<0.001)$, whereas in between groups comparison, the results were significant $(p<0.05)$ at 4 weeks, very significant $(\mathrm{p}<0.01)$ at 8 weeks, and highly significant $(\mathrm{p}<0.001)$ at 12 weeks. This indicated that add-on therapy of $N$. sativa oil leads to more prominent increase in eGFR than conservative management with KAA alone. Previous studies also demonstrated that ketoanalogs supplementation exhibited enhancement in GFR in CKD patients (Stages III and IV) [25]. It was also witnessed that administration of $N$. sativa oil at a dose of $1000 \mathrm{mg} / \mathrm{kg}$ body weight/day for 8 weeks caused restoration of renal hemodynamic and functions in streptozotocininduced diabetic rats in the form of increased GFR and effective renal blood flow [26]. The add-on effect might be due to reason that both KAA and $N$. sativa oil acted through different mechanisms.

There are only few studies with KAA in patients of DN, while no comparative study of KAA (control group), with $N$. sativa oil as an add-on therapy in addition to KAA (test group), in the conservative management of CKD KAA has been reported till date.

Some researchers postulated that KAA are transaminated by taking nitrogen from non-essential amino acids, thereby decreasing the formation of urea by re-using the amino group leading to decrease in levels of uremic toxins [27]. It was also been reported that keto-acids tend to reduce protein degradation and urinary protein excretion, and cause reduction of plasma urea, serum creatinine and improved eGFR in patients of CKD Stages 3 and 4 [22].

Further, it has been reported that there is significant reduction in TNF- $\alpha$, CRP, and adiponectin on keto acid supplementation in type 2 DN [28]. These plausible mechanisms might be the reason for beneficial effects of KAA in our present study.

However, earlier studies have also described the favorable effects of $N$. sativa regarding renal functions [9,24], hepatic functions [9], blood glucose level [29], blood pressure level [30], and lipid profile [31]. It was suggested that thymoquinone, an active ingredient of $N$. sativa oil, has strong antioxidant and anti-inflammatory properties and studies have provided original observations on the role of oxidative stress and inflammation in development of various renal diseases [32]. Our present study also demonstrated favorable effects on RFT parameters, which might be due to strong antioxidant and anti-inflammatory effect of thymoquinone.

The dose of KAA in previous clinical studies was $600 \mathrm{mg}$ TDS [22]. The doses of $N$. sativa in other clinical studies were $2.5 \mathrm{ml}$ oil twice daily [33], $3 \mathrm{~g}$ oil/day [34], and $2 \mathrm{~g}$ powder of $N$. sativa seeds/day [33]. Hence, the dose of $N$. sativa oil, we selected in our study was $2.5 \mathrm{ml} /$ day, which lies between minimum and maximum mentioned doses above and also due to compromised renal status in patients of CKD.

From the correlation table, it was evident that with the decrement in urea there was progressive increase of eGFR which was significant thereby implicating a negative correlation, which is significant thereby implicating that as the urea decreased the GFR improved, the same was observed with urea and creatinine where positive correlation was observed which was statistically highly significant, showing that as urea decreased so is creatinine, showing that both these parameters are complementary to each other in the prognosis of CKD.

On the basis of severity classification, the ADRs were mild in most of the cases (no hospitalization, no change of therapy, and no additional treatment required). All the ADRs were of latent or sub-acute in onset on the basis of onset of action, and no case belonged to the category of acute onset (within $60 \mathrm{~min}$ ). The adverse drug events were possible in 2 cases and probable in 1 case in control group (KAA), and possible in 5 cases, and probable in 1 case in test group (N. sativa oil supplemented group along with KAA) on the basis of Naranjo's Scale. Previous studies on N. sativa did not find any serious adverse effects, except few cases who experienced mild nausea in the beginning which finally disappeared in the $2^{\text {nd }}$ week of study [35]. Hence, the ADRs in both groups might be due to the manifestations of underlying renal pathology or probably due to other coadministered drugs.

\section{CONCLUSION}

N. sativa oil supplementation at a dose of $2.5 \mathrm{ml}$, p.o., once daily in addition to KAA (600 mg tablet) 3 times a day, along with conservative management was found to be safer for use as an add-on therapy; A significant improvement in the $\mathrm{Hg} \%$ and renal functions in patients of DN suggested that oil of $N$. sativa can be taken as an add-on therapy once a day along with KAA tab 3 times a day.

It has been also proved that both drugs are acting by a different mechanism, as N. sativa was showing its add-on effects, in addition to effects shown by KAA. However, exact mechanism regarding its efficacy needs to be explored.

The doses of $N$. sativa in the present study were $2.5 \mathrm{ml} /$ day which lies between maximum and minimum of doses taken in the previous studies, we have chosen this dose as there were studies with the abovementioned dose, and since we were giving this drug as an add-on we wanted to avoid drug toxicity.

We also found out a negative correlation between urea and eGFR, thereby implicating that the drug has an effect on both parameters simultaneously and as urea levels decreased the eGFR improved significantly, and also positive correlation between urea and creatinine suggested that both parameters increase or decrease simultaneously, and both deal with the prognosis of CKD.

\section{AUTHORS' CONTRIBUTIONS}

Study conception and design were done by Dr. F Haque and Dr. Rahat Ali Khan, Acquisition of data, analysis, and interpretation of data was done by Dr. Mohd Ashraf Alam. Drafting of manuscript and review was done by Dr. Mohammad Nasiruddin. Critical revision and organization of manuscript were done by Dr. Rahat Ali Khan. 


\section{CONFLICTS OF INTEREST}

The authors have no conflicts of interest.

\section{REFERENCES}

1. World Health Organization. Diabetes. Geneva: World Health Organization; 2015.

2. International Diabetes Federation. IDF Diabetes Atlas. $6^{\text {th }}$ ed. Brussels: International Diabetes Federation; 2014.

3. Lim AK. Diabetic nephropathy - complications and treatment. Int $\mathbf{J}$ Nephrol Renovasc Dis 2014;7:361-81.

4. Cheng W, Hua S, Chun Z. Role of NADPH oxidase in metabolic diseaserelated renal injury: An update. Oxid Med Cell Longev 2016;2016:1-8.

5. Hojs R, Ekart R, Bevc S, Hojs N. Markers of inflammation and oxidative stress in the development and progression of renal disease in diabetic patients. Nephron 2016;133:159-62.

6. Kher V. End-stage renal disease in developing countries. Kidney Int 2002;62:350-62.

7. Qiu HY, Liu F, Zhao LJ, Huang SM, Zuo C, Zhong H, et al. Comparison of the effects of alpha-keto/amino acid supplemented low protein diet and diabetes diet in patients with diabetic nephropathy. Sichuan Da Xue Xue Bao Yi Xue Ban 2012;43:425-8.

8. Prakash S, Pande DP, Sharma S, Sharma D, Bal CS, Kulkarni H, et al. Randomized, double-blind, placebo-controlled trial to evaluate efficacy of ketodiet in predialytic chronic renal failure. J Ren Nutr 2004;14:89-96.

9. El-Shamy KA, Mosa MM, El-Nabarawy SK, El-Qattan GM. Effect of Nigella sativa tea in Type 2-diabetic patients as regards glucose homeostasis, liver and kidney functions. J Appl Sci Res 2011;7:2524-34.

10. Mohtashami R, Amini M, Huseini HF, Ghamarchehre M, Sadeqhi Z, Hajiagaee R, et al. Blood glucose lowering effects of Nigella sativa L. seeds oil in healthy volunteers: A randomized, double-blind, placebo controlled clinical trial. J Med Plants 2011;10:90-4.

11. Martínez-Castelao A, Navarro-González JF, Górriz JL, de Alvaro F. The concept and the epidemiology of diabetic nephropathy have changed in recent years. J Clin Med 2015;4:1207-16.

12. Gheith O, Farouk N, Nampoory N, Halim MA, Al-Otaibi T. Diabetic kidney disease: World wide difference of prevalence and risk factors. J Nephropharmacol 2016;5:49-56.

13. Lipska KJ, Krumholz H, Soones T, Lee SJ. Polypharmacy in the aging patient: A review of glycemic control in older adults with Type 2 diabetes. JAMA 2016;315:1034-45.

14. Schwartz AV, Vittinghoff E, Sellmeyer DE, Feingold KR, de Rekeneire $\mathrm{N}$, Strotmeyer ES, et al. Diabetes-related complications, glycemic control, and falls in older adults. Diabetes Care 2008;31:391-6.

15. Shurraw S, Hemmelgarn B, Lin M, Majumdar SR, Klarenbach S, Manns B, et al. Association between glycemic control and adverse outcomes in people with diabetes mellitus and chronic kidney disease: A population-based cohort study. Arch Intern Med 2011;171:1920-7.

16. Ricks J, Molnar MZ, Kovesdy CP, Shah A, Nissenson AR, Williams M, et al. Glycemic control and cardiovascular mortality in hemodialysis patients with diabetes: A 6-year cohort study. Diabetes 2012;61:708-15.

17. Wild S, Roglic G, Green A, Sicree R, King H. Global prevalence of diabetes: Estimates for the year 2000 and projections for 2030. Diabetes Care 2004;27:1047-53.
18. Gupta R. Trends in hypertension epidemiology in India. J Hum Hypertens 2004;18:73-8.

19. Jha V.ESRD in India and Pakistan 2013. Kidney Int Suppl2013;3:157-60.

20. Jeloka TK, Upase S, Chitikeshi S. Monthly cost of three exchanges a day peritoneal dialysis is same as of thrice a week hemodialysis in selfpaying Indian patients. Indian J Nephrol 2012;22:39-41

21. Jha V, Garcia-Garcia G, Iseki K, Li Z, Naicker S, Plattner B, et al. Chronic kidney disease: Global dimension and perspectives. Lancet 2013;382:260-72.

22. Khan IA, Nasiruddin M, Haque SF, Khan RA. A randomized clinical trial to evaluate the efficacy and safety of $\alpha$-keto amino acids supplementation in stage 3 and 4 patients of chronic kidney disease. Asian J Pharm Clin Res 2014;7:21-4.

23. Sayed-Ahmed MM, Nagi MN. Thymoquinone supplementation prevents the development of gentamicin-induced acute renal toxicity in rats. Clin Exp Pharmacol Physiol 2007;34:399-405.

24. Ansari ZM, Nasiruddin M, Haque SF, Khan RA. Evaluation of efficacy and safety of Nigella sativa oil supplementation in patients of chronic kidney disease. Asian J Pharm Clin Res 2016;9:107-10.

25. Chang JH, Kim DK, Park JT, Kang EW, Yoo TH, Kim BS, et al. Influence of ketoanalogs supplementation on the progression in chronic kidney disease patients who had training on low-protein diet. Nephrology (Carlton) 2009;14:750-7.

26. Yusuksawad M, Chaiyabutr N. Restoration of renal hemodynamics and functions during black cumin (Nigella sativa) administration in streptozotocin-induced diabetic rats. J Exp Pharmacol 2012;4:1-7.

27. Teplan V. Supplements of keto acids in patients with chronic renal failure. Nefrol Derg 2004;13:3-7.

28. Chen N, Jin Y, Ren H, Jing X, Shen P, Huang X. Anti-inflammatory effects of low protein diet supplemented with keto-amino acid in the treatment of Type 2 diabetic nephropathy. Kidney Res Clin Pract 2012;31:A24.

29. Bamosa AO, Kaatabi H, Lebdaa FM, Elq AM, Al-Sultanb A. Effect of Nigella sativa seeds on the glycemic control of patients with Type 2 diabetes mellitus. Indian J Physiol Pharmacol 2010;54:344-54.

30. Dehkordi FR, Kamkhah AF. Antihypertensive effect of Nigella sativa seed extract in patients with mild hypertension. Fundam Clin Pharmacol 2008;22:447-52

31. Kaatabi H, Bamosa AO, Lebda FM, Al Elq AH, Al-Sultan AI. Favorable impact of Nigella sativa seeds on lipid profile in Type 2 diabetic patients. J Family Community Med 2012;19:155-61.

32. Ragheb A, Attia A, Eldin WS, Elbarbry F, Gazarin S, Shoker A, et al. The protective effect of thymoquinone, an anti-oxidant and antiinflammatory agent, against renal injury: A review. Saudi J Kidney Dis Transpl 2009;20:741-52.

33. Najmi A, Nasiruddin M, Khan RA, Haque SF. Effect of Nigella sativa oil on various clinical and biochemical parameters of insulin resistance syndrome. Int J Diabetes Dev Ctries 2008;28:11-4.

34. Heshmati J, Namazi N, Memarzadeh MR, Taghizadeh M, Kolahdooz F. Nigella sativa oil affects glucose metabolism and lipid concentrations in patients with Type 2 diabetes: A randomized, double-blind, placebocontrolled trial. Food Res Int 2015;70:87-93.

35. Fallah Huseini H, Amini M, Mohtashami R, Ghamarchehre ME, Sadeqhi Z, Kianbakht S, et al. Blood pressure lowering effect of Nigella sativa L. Seed oil in healthy volunteers: A randomized, double-blind, placebo-controlled clinical trial. Phytother Res 2013;27:1849-53. 